\title{
Tailoring Lifelong Cultural Experiences
}

Jacqueline Floch ${ }^{1}$, Shanshan Jiang ${ }^{1}$, Maria Eugenia Beltrán ${ }^{2}$, Eurydice Georganteli ${ }^{3}$, Ioanna N. Koukouni ${ }^{3}$, Belén Prados ${ }^{4}$, Lucia María Pérez ${ }^{4}$, María del Mar Villafranca ${ }^{4}$, Silvia de los Ríos ${ }^{5}$, María F. Cabrera-Umpiérrez ${ }^{5}$, and María T. Arredondo ${ }^{5}$

\author{
${ }^{1}$ SINTEF ICT, N-7465 Trondheim, Norway \\ \{jacqueline.floch, shanshan.jiang\} asintef.no \\ ${ }^{2}$ INMARK Estudios y Estrategias, Spain \\ xenia.beltran@grupoinmark. com \\ ${ }^{3}$ University of Birmingham, United Kingdom \\ \{e.georganteli,i.koukounis\} @bham.ac.uk \\ ${ }^{4}$ Patronato de la Alhambra y Generalife, Spain \\ belenprados@wonderbrand.es, \\ \{luciam.perez, mariamar.villafranca\} ajuntadeandalucia.es \\ ${ }^{5}$ Life Supporting Technologies, Universidad Politécnica de Madrid, Spain \\ \{srios, chiqui, mta\} alst.tfo.upm.es
}

\begin{abstract}
ICT-based personalization in cultural heritage has been an important topic of research during the last twenty years. Personalization is used as a means to enhance the visitors' experience of a cultural site. Little consideration has however been set on lifelong cultural experiences, i.e. engaging the public in culture beyond the visit of a single site and bridging multiple sites. Cultural sites differ leading to a diversity of needs that should be taken into account through a personalization approach. This paper presents a set of scenarios tailored to suit the needs of three different Cultural Heritage sites in different EU countries. These scenarios have been developed within the EU funded project TAG CLOUD that aims at leveraging existing technologies to support realistic lifelong engagement experiences with cultural heritage through personalized content and interaction.
\end{abstract}

\section{Introduction}

Despite a rich European cultural heritage and the deployment of novel exhibition approaches and tools in museums and cultural institutions, the public engagement in cultural heritage remains low. According to recent statistics, 55\% of the European population did not visit any cultural site in 2010 [1]. The EU funded project TAG CLOUD addresses the challenge of engaging a global audience with cultural heritage. To that aim, the project devises a set of applications built upon a personalization framework. The approach will enable the support of multiple forms of interaction with culture in multiple cultural sites. Personalization is thus envisaged at two levels: both contents and interactions can be adapted to individual user profiles. We use the term "interaction" in a broad sense to depict different dissemination goals, e.g. storytelling and gamification, 
different augmented modules, e.g. information layers and avatars, and different social interactions, e.g. commenting and sharing.

Personalization in cultural heritage is not new. Much research has been conducted in the field during the last twenty years, but no satisfactory solution has yet been achieved. According to Ardissono et al., a major issue is the lack of realistic scenarios and experiments that put research works into practice [2]. This is the strategy adopted by TAG CLOUD. Rather than developing new advanced technologies, we wish to leverage existing technologies and cultural databases, and to combine them in order to create an experimental platform.

Beyond the variety of visitors, the diversity of needs of the cultural sites should also be taken into account by a personalization approach. This is illustrated in this paper through the presentation of a set of selected scenarios tailored to suit the needs of the three cultural pilot sites involved in TAG CLOUD. Interestingly, the result of the scenario design work is that the needs expressed by the pilot sites through the scenarios acknowledge the new opportunities for personalization identified in the survey presented in [2], i.e. adaptive cultural experience, social experience and user generated content, as well as benefits from individualized support that takes into account contextual and personal attributes.

TAG CLOUD scenarios illustrate different contexts of use, such as location (i.e., off-site or on-site) and time (i.e., before, during or after a visit), and different users (i.e. cultural users, non-cultural users and professionals). The scenarios also illustrate different use cases: user and group management, user profile management, cultural artefact management, itinerary management, interaction though virtual environments, storytelling, social behavior, content search and recommendation, and content adaptation. Due to space limitations, the paper concentrates on one selected scenario per pilot site. The selected scenarios relate to the new opportunities depicted in [2]: The Monumental Complex of Alhambra and Generalife focuses on adaptive cultural experience, the Barber Institute of Fine Arts on group experience and the County of Sør Trøndelag on user-generated content.

The rest of the paper is organized as follows: Section 2 shows the different settings and needs of the pilot sites, Section 3 describes the methodology for scenario design, Sections 4-6 present the selected scenarios from the three pilot sites and finally Section 7 gives the evaluation results obtained so far and outlines the road ahead.

\section{The Pilot Sites}

TAG CLOUD involves three cultural pilot sites: the Monumental Complex of Alhambra and Generalife in Spain, the Barber Institute of Fine Arts at the University of Birmingham in United Kingdom, and the County of Sør Trøndelag in Norway. The reason for including three sites is twofold: On one hand, the pilot sites present different characteristics and raise different requirements to the project. On another hand, we wish to provide solutions for lifelong experiences rather than focusing on experiences in a single site. 
The Monumental Complex of Alhambra and Generalife is a major European cultural site that attracts more than 3 Millions of visitors each year. In this case, the main goal is not to increase the number of visitors but rather to explore different interaction modes in order to provide an enhanced adapted experience to different users and to offer a varied repertory of theme-based itineraries. Such itineraries will include internal and external routes that support new ways of enjoying the urban and country environment of the Alhambra. Another concern is also that of engaging users in other cultural activities after their visit in Alhambra, thus facilitating a diversification of the tourism and cultural offer. A technical challenge in the pilot site is the combination of an indoor and outdoor visit.

The Barber Institute of Fine Arts, the "mini National Gallery of Britain" in the West Midlands, United Kingdom, is an art collection and gallery at the University of Birmingham. It houses a superb collection of works of art, among which the finest collection of Byzantine coins worldwide. Seals, Roman, and medieval Islamic and western coins supplement the collection. Only a fraction of the Barber Institute's 16,000 coins and seals are on permanent display (20-30), whilst another 100-200 are showcased on a rolling base in a state-of-the-art Coin Gallery. Through its participation in the TAG CLOUD project the Barber Institute aims to converse more effectively with its core audience, and to reach out to a global audience, furthering social engagement and alerting its diverse audience of the endless possibilities cultural heritage provides for the exploration of interfaith and intercultural dialogue.

The third pilot site relates to the development of digital cultural heritage in the landscape, more specifically in the County of Sør Trøndelag. In that case, the cultural artefacts are places, e.g., buildings, heritage sites or art in the landscape. They do not always lie under the responsibility of a cultural institution and thus may not yet be registered and catalogued in databases. In addition, as there are a large number of artefacts, it is not always possible to rely on the curators or other professionals for registration and storytelling due to personnel resource limitations. A main goal is to increase participatory approach and engage the community in discovering and telling stories about places they live in and are fond of.

\section{A User-Centered Approach}

In addition to the diversity of sites, the project fosters a user-centered design approach. At an early stage of the project, the pilots have contributed to the definition of realistic scenarios that fit their environments and needs. These scenarios were evaluated by potential users and by professionals in the cultural sector.

Scenario building is a widely accepted way to generate design ideas for new systems and products and to identify the possible users and contexts of use for these systems and products. Scenarios describe individual users in individual usage situations and are not meant to describe the whole system functionality. The value of scenarios is that they concretize something for the purpose of analysis and communication [3-4]. In particular, user-oriented design emphasizes representing the use of a system or application with a set of user interaction scenarios as these make the use of the 
system (or application) explicit, and make it easy to evaluate and document a given design decision in terms of the specific consequences within defined scenarios [4]. Use cases describe interactions with the system at a more detailed level than scenarios. When a scenario-based approach is used, the use cases are normally extracted from the scenarios. Use cases then serve as functional requirements to the system from the stakeholder viewpoint.

The TAG CLOUD approach to scenario and use case specification has been derived after reviewing state-of-the-art methodologies on scenario design [5-7]. We define an overall methodology consisting of the following main steps and each pilot site has refined the process according to the special needs of the sites.

1) Scenario brainstorming: Stories and scenes were produced illustrating the system features. The partners were organized in three clusters built around the three trial sites in United Kingdom, Spain and Norway. In this stage the user needs and pilot site expectations had been identified through user surveys carried out either by pilot sites, e.g. visitor surveys in United Kingdom and Alhambra, or by others, e.g., available surveys about cultural participation in Norway [8-10] and Denmark [11]. These served as an input for brainstorming. Thus, TAG CLOUD could be used in multiple sites through Europe.

2) Internal feedback and scenario revision: The scenarios were reviewed by partners from other trial sites and revised. To give better understanding of the features a set of generic scenarios were defined, which cover the scenarios proposed for each trial site, but are not dependent on the cultural contents specific to the trial sites.

3) Use cases identification and description: Use cases were identified for describing the interaction of the users with the system at a more detailed level than the scenarios. The same use cases were often extracted from multiple scenarios, e.g. system logging and sharing on Facebook. Therefore the use cases were first extracted and classified, before being described in more details.

4) User evaluation: The scenarios were evaluated by potential users (the public and professionals in the cultural domain). This step can be performed before or in parallel with the previous step. The evaluation was conducted at different points of time and using different approaches at the different trial sites depending on the needs of the sites. For instance, in Norway where the target user groups for the evaluation of TAG CLOUD solutions are secondary school students, the scenarios were evaluated in the spring before the school holidays, and before the identification of use cases. In addition, different evaluation methods were used, including user surveys, workshops and focus group with both end users and professionals. For instance, exploratory focus groups [12-13] with school students and professionals from the culture, tourism and IT sectors were organized to evaluate scenarios in Norway. Protocols for focus group including question routes were designed following the methodology and guide-lines from literature [14]. Storyboards [15] were prepared for presenting the scenarios in focus groups, also allowing us to identify unclear points in the scenarios.

5) Scenarios and use cases finalization: The scenarios were refined based on the feedback from the user evaluation. 


\section{$4 \quad$ Scenario 1: Adaptive Cultural Experience}

"Tour at the Palace of the Lions: Enjoying an adaptive cultural experience in a heritage institution" is a scenario that has been developed and adapted to the specificities of the Monumental Complex of Alhambra and Generalife. This scenario presents how the Alhambra aims at enhancing the visitors' cultural experience by integrating augmented reality and storytelling techniques, as well as supporting a better understanding of this Monumental Complex and its cultural value to different type of visitors, by using personalization and adaptation embedded capabilities.

Hence, this scenario emphasizes, among others things, on how the TAG CLOUD App will provide personalized multimedia content and information to small heterogeneous groups (e.g., families), through different ways of interaction and using augmented reality to enrich the architectural remains and cultural value of the Alhambra Monument Complex and its Palace City context, with information that would be otherwise not perceivable. The scenario also illustrates the adaptation and personalization approach by displaying information in different formats and with significant differences depending on the user's profile.

The scenario's main character, Martin, is an architect who visits the Monumental Complex of Alhambra and Generalife with his two children. Martin is especially interested in how the Fountain Court of the Lions, placed within the Monumental Complex, has been restored, its complexity, and the large number of studies that have been done around it; as the restoration of the Fountain Court of the Lions won the prize of conservation Europa Nostra in 2013. But also, Martin wants that his children John (8Y) and Mary (6Y) also enjoy the visit.

Therefore, the visit will be done using the TAG CLOUD App. Martin and his children already have an account in TAG CLOUD, and can log into the TAG CLOUD App through different devices. When they arrive to the Monumental Complex of Alhambra and Generalife, Martin rents a mobile device (tablet), John uses his iPad and Mary her smartphone.

At the entrance, the App shows each member of the family a list places in Alhambra that relate to their interests. Martin is shown a list about architectural conservation and restoration. Along the route, the TAG CLOUD App guides him through the points of interest and hints him that he can use Augmented Reality (AR) when pointing different buildings and places with the mobile device camera. The AR mode superposes information on the image scanned by the mobile device, such as the direction to follow, the suggested interesting points of interest and information related to them. In the AR mode, the TAG CLOUD App shows an icon related to each point of interest on the tablet screen. Martin only has to touch an icon to access information about a point of his interest, and then can receive personalized information. In addition, the App provides access to stories about the sites through the storytelling tool.

When Martin arrives to the Court of the Lions the App displays various kinds of information related to the Court of the Lions, e.g., about the heritage landmark and about the restoration, and in different information modes, e.g., text, photo and video. TAG CLOUD allows a deeper understanding to restoration process with the combination of documentation and audiovisual exhibitions through the storytelling tool, such 
as the documentation associated with the exhibition about the restoration process of the first lion. Also, TAG CLOUD gives access to videos from visitors on the Court of the Lions and from the media that match with Martin's interests.

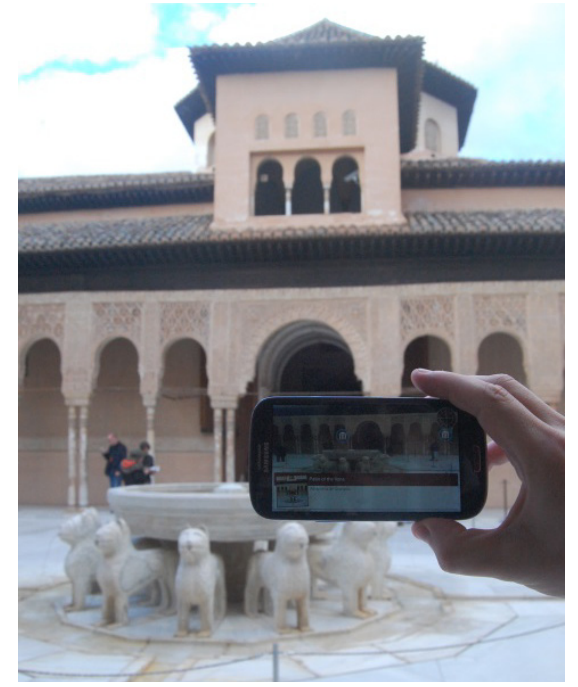

Fig. 1. Augmented Reality in the Court of the Lions

While Martin enhances his knowledge of the Court of the Lions and enjoys his visit, his children get the suggestion to use the game of the Court of the Lions. They start the game and have fun painting directly on their iPad and smartphone, choosing a lion to paint and colour.

After the visit, when Martin and his children return home, Martin starts participating in one of the group of interest of the Monumental Complex of Alhambra and Generalife. One of the members of this group recommends him some publications. The children continue playing at home with the different games offered by the web of the Alhambra through TAG CLOUD and they can also share pictures from their visit with their Facebook friends, as well as details of the places that they have discovered through the games. In this way, the family continues enjoying culture after visiting the Monumental Complex of the Alhambra.

Through the enriched cultural experience like the one illustrated in this scenario, the Monumental Complex of Alhambra and Generalife expects to foster engagement and boost the spreading of word of mouth. In the future, John and Mary will ask their father to visit the Alhambra again, go on their own and with their own families.

\section{Scenario 2: Group Experience}

In the context of TAG CLOUD the University of Birmingham chose to develop the theme "Along Europe's Cultural Routes", as it provides an excellent platform for 
visitors to explore important themes such as shared heritage, identity and citizenship. The theme can greatly impact on a very large audience in Europe and beyond.

Use of mobile devices is very popular nowadays among all age groups [16]. We find it intriguing to imagine how a group of visitors with similar interests or objectives (e.g., students, mature visitors doing a guided tour or school children) will respond to a novel system applied on a cultural site.

In the context of their course on Medieval Archaeology - Creating Europe: Complex Societies 1000 BC - AD 1000, students will be visiting the Barber Institute Coin Collection to handle and discuss medieval coins and compare them with other contemporary works of art from the Barber Institute Collections.

Prior to their visit to the Barber Institute, their academic tutor Lydia, has prepared the visit in the seminar room by alerting students to the TAG CLOUD App downloadable from the Barber website. She discusses with them a virtual itinerary for the visit and makes quizzes related to the visit. The students register to TAG CLOUD via the museum's web page and the App leads them to the virtual itinerary entitled "Along Europe's Cultural Routes" customized by Lydia.

One of the journeys begins with a splendid Byzantine gold solidus from the Coin Collection of the Barber Institute of Fine Arts. A virtual historical character (in this case the Byzantine emperor depicted on the obverse of the coin) narrates the story of the coin from Constantinople/Istanbul, where it was minted, all the way to Durrachium/Durrazo/Durrës on the Adriatic Sea and along the Via Egnatia, one of Europe's major and oldest cultural routes. The students follow the coin's journey, exploring medieval cities, major landmarks and related artefacts along that route, and deepening their understanding of urban life in the Middle Ages.

Other carefully selected coins from the Barber Institute Collection allow them to follow other important European cultural routes, namely the ones linking Byzantium with Anglo-Saxon Britain, Byzantium with Norway (Scandinavia), and Byzantium with medieval Spain.

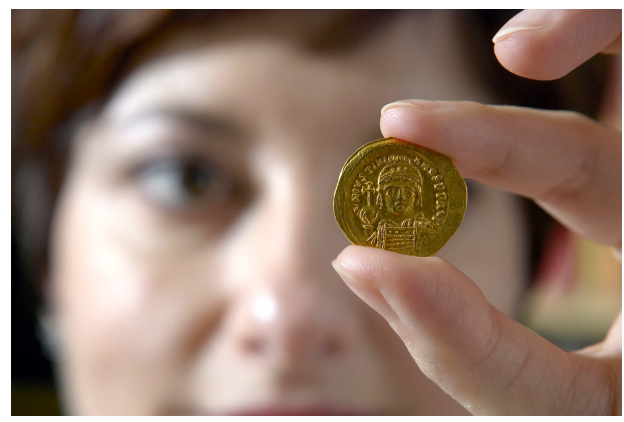

Fig. 2. Up close and personal with life in medieval Europe: Byzantine gold solidus of Emperor Justinian I (527-565) from the Barber Institute Coin Collection

For the visit, tablets are provided by the University of Birmingham Digital Humanities Hub. The tablets have the same group code so that students embark on the same itinerary. On-site visits offer students the experience of handling coins and 
understanding them in the context of the medieval and early Renaissance section of the Barber Institute Collections. TAG CLOUD provides handy information with links and images for handling coins and carrying out the task of comparison. Through enquiry-based learning, quizzes, online games, puzzles, and close-up viewing and handling of select Barber Institute artefacts students are at the centre of learning process, understanding the Middle Ages as a highly interconnected world.

After their visit the students discuss their experience and write their stories and impressions using TAG CLOUD collaborative storytelling tool, where they can find the participation of fellow students and experts in the field that enrich their story. They also share their stories via social networks.

This scenario emphasizes the creation of group experience adapted to groups with similar needs through customised group itineraries, and the social interactions within the group through quizzes and games.

Visitors follow groups' personalized itineraries through time and space; the historical characters-guides, who are being developed for the needs of the four itineraries, can bring history to life and engage visitors with aspects of European cultural heritage, tangible and intangible. The interaction design of the Apps is easy to use (www; digital images; geo-references; graphics), making the information components appealing to visitors of different age groups.

Along each route visitors see real-life images of monumental architecture, paired with appropriate coins and select 3D and AR visualizations of historical topography and artefacts. Geo-spatial information and selected historical sources enhance significantly the Barber Institute experience and aim to put visitors at the centre of learning process through storytelling and attractive virtual journeys, and to alert them of Europe's shared heritage, superbly showcased in the messages and iconography displayed on medieval coins.

\section{$6 \quad$ Scenario 3: User-Generated Content}

This scenario is inspired by the initiative Væggen by the Museum of Copenhagen ${ }^{1}$. Væggen is a large multitouch screen allowing people to tell, share and experience stories. This scenario explores the concept of wall as means to discover and share cultural stories related to buildings and heritage places in city open spaces. It is however not practical to install and maintain physical walls such as Væggen all around. Instead we propose a digital representation of walls, that we call Virtual Wall.

The storyline of the scenario is as follows. Kjersti, a research librarian, decides to create and share virtual walls for her home city Trondheim. When she launches the Virtual Wall through the TAG CLOUD App, a map of the city marked with available walls is shown. Since no one has created a wall for the cathedral, she positions the mouse pointer to the front of the cathedral and selects the creation button. Then she uploads a picture of the West Front. The wall is automatically tagged with the location selected by her and the creator name. She annotates the wall by selecting among

1 http: //vaeggen. copenhagen. dk/en/. Væggen is the Danish word for Wall. 
tags suggested by the system or creating new tags. She then opens the project allowing others to add annotations.

Kjersti has selected some of the 57 west front sculptures for annotation. She zooms in/out the wall picture and adds annotations by clicking on the sculpture in the picture and adding information. She can provide a name and a description consisting of text and multimedia links. She can also add predefined or new tags to her annotations.

She shares the wall with her friends through Facebook and gets a lot of responses. Her Facebook friends have added "likes" to her annotations and commented. Her friend Kjell-Jørgen has added a timeline to the wall and annotated dates with references to some old pictures he found on websites. Øystein, an archaeologist working at the restoration workshop, also reviews the work and adds overall information to the wall.

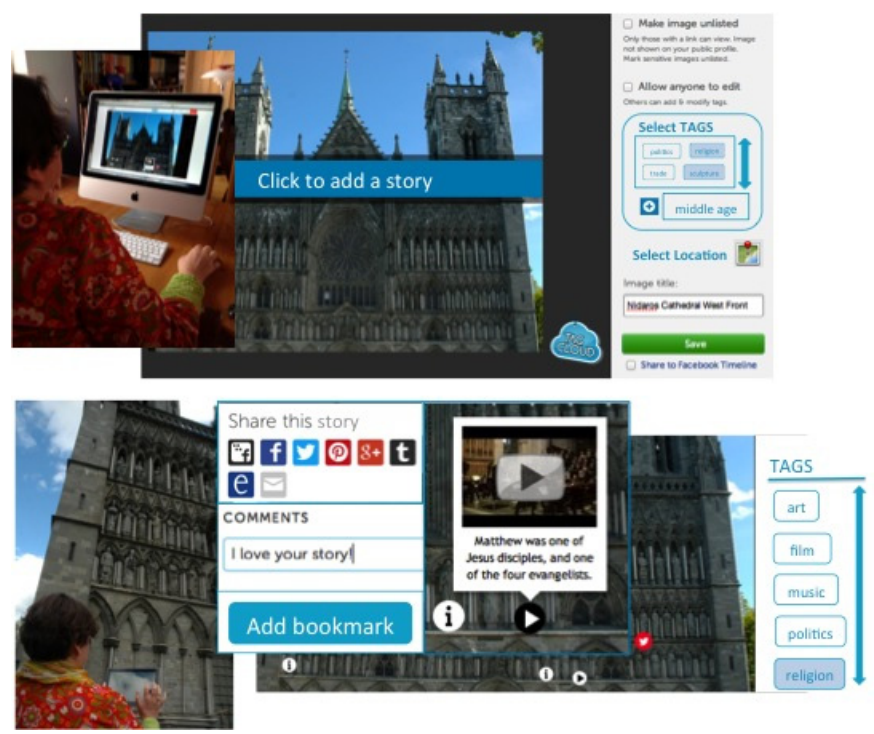

Fig. 3. Virtual Wall and storytelling: creating stories at home and experiencing on site

On his first visit to the cathedral, Paul can easily retrieve the wall and the stories using the Virtual Wall on TAG CLOUD App on his smart phones. Both location- and tag- based search is available. The "personalize" feature highlights his personal tags. He selects "music" and finds that no story is under this tag. He remembers that his grandfather told him that a sculptor of statue of the Archangel Michael was inspired by musician Bob Dylan, so he adds the story and shares with Facebook.

The Virtual Wall demonstrates important features of adaptive and social storytelling, e.g., retrieval of wall and filtering stories according to location, popularity, topics and other metadata (e.g. author, duration), sharing walls and stories for editing and commenting, as well as sharing walls and stories through social networks. Its novelty lies in allowing users to generate contents, share with others for commenting and collaborative editing, as well as the presentation on map and timeline. Moreover, the 
Virtual Wall aims at having an active participation of the Trondheim community and visitors, supporting sustainable use and knowledge of the city`s cultural heritage and provide knowledge through several interpretations of cultural heritage, identity, and voices for the different city spaces by their community and visitors.

\section{$7 \quad$ Status and Further Work}

Two initial evaluations were conducted. Both potential users and cultural heritage managers were involved. The first evaluation organized in Norway had focus on adaptive and social storytelling. The second organized in Spain had focus on adaptive content and interaction.

In Norway, evaluation was conducted through focus groups. Four focus groups involving students from two different secondary schools and two focus groups involving culture professionals were organized. Generally students were very positive to the ideas presented while the response from professionals was more mixed. Among all feedback, we retain here the three most important points: 1) Trustworthiness of usergenerated content. As the Virtual Wall was presented as a means to let any person to contribute with stories, it is important to maintain a high quality of contents. This applies to the correctness of content and tags associated to them. Descriptions from professionals are generally considered to be trustworthy. A mixed approach of contents created by professionals and amateurs is suggested. Providing references and sources contributes to increased trustworthiness. Filtering and being able to report spam is essential with respect to quality. In addition, support for access control is needed for collaborative editing to retain credibility. 2) The importance of social behavior. The students wish to share with friends, give them hint. They also wish to follow friends, e.g. where they have visited and what they have commented. The public can contribute positively to the personalisation and promotion of contents: "not only that the West Front exists, but I've been there". Not all would contribute with creation of stories, but there are many ways of contributions, for example, review and commenting. 3) Support for personal exploration. It is essential to have quality-based search, filtering and personalized recommendation: "Not only what others do, but what $I$ may like". User history and different needs in different contexts should be considered for recommendation.

During the evaluation in Spain, participants recognized that nowadays there are more and more technological initiatives towards personalization of Cultural Heritage sites experiences, however these are scattered. Participants also agreed that these experiences depend on the physical, personal, and socio-cultural context, and identityrelated aspects. Hence participants acknowledged that they can benefit from individualized support that takes into account contextual and personal attributes, ongoing adaptation offsite and "on the go" as visitors' behavior may not remain consistent during a visit and new opportunities to capture and share the "whole story" (e.g. history, stories and culture) in a meaningful way. Participants affirmed that when the users' background and preferences are recognized and incorporated into the process of communicating the wealth of cultural heritage sites, the cultural experience will 
empower visitors' motivation and engagement for culture; specially young people. Cultural heritage sites' managers commented that they have the need of efficient solutions to manage all created digital content to be used in a user-centric and targeted way; this process is seen as a labor intensive and costly activity. Thus the challenge that needs to be overcome by large and small cultural heritage sites derives from the growing interest in supporting socialization and collaboration in small (e.g., archaeologists) and large-size communities (e.g., schools) and the interest in user-generated content, coupled with a need to guarantee high-quality information standards at low costs. Participants saw the scenarios provided in TAG CLOUD could take place in 58 years.

After the evaluations, a set of initial prototypes that concretize some important features of the scenarios were created. Based on these prototypes, that allowed us to identify practical implementation concerns, an initial selection of the features of the TAG CLOUD system, including cloud services and applications, was made. A set of final use cases was extracted from both the scenarios and initial prototypes. These prototypes will be further extended to realize these use cases.

As a next step in our work, a preliminary laboratory testing of Low/Medium Fidelity prototypes will be performed before the pilot trials that will be organized at each site. The aim is to collect feedback about TAG CLOUD solutions from both visitors and cultural institutions.

Acknowledgement. This work is partially funded by the EC FP7 project TAG CLOUD (Technologies lead to Adaptability \& lifelong enGagement with culture throughout the CLOUD), G.A. No. 600924. http://www.tagcloudproject.eu/

\section{References}

1. Eurostat: Cultural statistic. Publications Office of the European Union (2011)

2. Ardissono, L., Kuflik, T., Petrelli, D.: Personalization in cultural heritage: the road travelled and the one ahead. User Modeling and User-Adapted Interaction 22(1-2), 73-99 (2012)

3. Carroll, J.M.: Scenario-based design: envisioning work and technology in system development. John Wiley \& Sons (1995)

4. Carroll, J.M.: Five reasons for scenario-based design. In: Interacting with Computers, vol 13, pp. 43-60. Elsevier (2000)

5. Di Nitto, E., et al.: S-Cube Deliverable CD-IA 2.2.2: Collection of Industrial Best Practices, Scenario and Business Cases. S-Cube consortium (2009)

6. Frank, K., et al.: PERSIST Deliverable 2.1: Scenario description and Requirements Specification. PERSIST consortium (2008)

7. Roussaki, I., et al.: SOCIETIES Deliverable 2.2: Scenario Description, Use Cases and Technical Requirements specification. PERSIST consortium (2011)

8. Bjørnsen, E., Lind, E., Hauge, E.S.: Kunstkonsum i storbyene - en studie av brukere og ikke-brukere av det offentlig finansierte kunsttilbudet i byene Oslo, Bergen, Trondheim, Stavanger og Kristiansand. FoU-rapport nr. 7/2012. Agderforskning (July 2012). Report available, http: / / norskpublikumsutvikling . no/2012/10/ kunstkonsum-i-storbyene (in Norwegian) 
9. Gran, A.-B., Wedde, E.: Publikum: Hvem, hva, hvorfor? Perduco Kultur. Report available at http://norskpublikumsutvikling.no/2012/05/for-lite-synligmuseum (in Norwegian)

10. Gran, A.-B., Figenschou, A., Gaustad, T., Molde, A.: Digitalt kulturkonsum- En norsk studie. Forskningsrapport (2012), Handelshøyskolen BI (2012). Report available at http: / / norskpublikumsutvikling.no/2012/12/digitaltkulturkonsum (in Norwegian)

11. Danish Agency for Culture: About the National User survey: http: / /www. kulturstyrelsen.dk/english/institutions / museums /museum-surveys (last access February 1, 2014)

12. Stewart, D.W., Shamdasani, P.N., Rook, D.W.: Focus Groups: Theory and Practice, 2nd edn., vol. 20. Sage Publications, Newbury Park (2007)

13. Tremblay, M.C., Hevner, A.R., Berndt, D.J.: The Use of Focus Groups in Design Science Research. In: Integrated Series in Information Systems, vol. 22, pp. 121-143. Springer, US (2010)

14. Krueger, R.A., Casey, M.A.: Focus Groups: A Practical Guide for Applied Research, 4th edn. Sage Publications, Thousand Oaks (2000)

15. Truong, K.N., Hayes, G.R., Abowd, G.D.: Storyboarding: an empirical determination of best practices and effective guidelines. In: Proceedings of the 6th Conference on Designing Interactive systems (DIS 2006). ACM, New York (2006)

16. Love, S.: Understanding mobile human computer interaction. Elsevier (2005) 